\title{
STABILITY IMPLICATIONS ON THE ASYMPTOTIC BEHAVIOR OF SECOND ORDER DIFFERENTIAL EQUATIONS
}

\author{
JOHN M. BOWNDS ${ }^{1}$
}

\begin{abstract}
Using some basic observations from stability theory, it is shown that the classical equation $y^{\prime \prime}+a(t) y=0$ must have at least one solution $y(t)$ such that $\lim \sup \left(|y(t)|+\left|y^{\prime}(t)\right|\right)>0$ as $t \rightarrow \infty$. The same conclusion holds for a nonlinear perturbation of this equation provided the linearization has a stable zero equilibrium. The results may be easily and naturally generalized to $n$th order equations, although that generalization is not done here.
\end{abstract}

1. Introduction. Consider the classical, canonical equation

$$
y^{\prime \prime}+a(t) y=0, \quad t \geqq t_{0},
$$

and a perturbation

$$
y^{\prime \prime}+a(t) y=f\left(t, y, y^{\prime}\right), \quad t \geqq t_{0} .
$$

Specifics on the assumptions for $a$ and $f$ will be made clear later.

Surely one of the standard problems concerning both (1.1) and (1.2) is that of determining conditions on $a(t)$ which imply that all solutions are asymptotic to zero. With regard to this problem for (1.1), reference is made to [1], [10], [12], [14], with more recent work found in [7] and [9]. Regarding (1.2), the literature is less massive; for typical results, see [2]-[5], [7], [8], [10], [11].

Another classical problem regarding (1.1) is that of determining the asymptotic behavior of solutions to (1.1) in terms of their first derivatives. A typical result along these lines is that certain growth requirements on $a(t)$ imply that any solution $y(t)$ to (1.1) has the properties that $|y(t)| \rightarrow 0$ and lim $\sup \left|y^{\prime}(t)\right|>0$ as $t \rightarrow \infty$. For example, an elegant theorem of Wiman [13] states that if

$$
W(t)=\lim _{t \rightarrow \infty} a^{\prime}(t)[a(t)]^{-3 / 2}=0,
$$

Received by the editors August 18, 1972.

AMS (MOS) subject classifications (1970). Primary 34D05, 34D10, 34C10; Secondary 34A30, 34C15, 34D20.

${ }^{1}$ This work supported by the Einstein Chair of Science, Rensselaer Polytechnic Institute, Troy, New York 12181

(C) American Mathematical Society 1973 
with $W(t)$ in $B V\left[t_{0}, \infty\right)$, then the quadratic form

$$
Q(t)=[a(t)]^{3 / 2}|y(t)|^{2}+[a(t)]^{-3 / 2}\left|y^{\prime}(t)\right|^{2}
$$

is bounded away from both zero and infinity.

It will be shown here (Theorem 1) that there always exists a solution $y(t)$ to (1.1) for which $\lim \sup |y(t)|+\left|y^{\prime}(t)\right|>0$ as $t \rightarrow \infty$. No growth requirements of any kind are needed for this result; it will only be assumed that $a$ is in $C\left[t_{0}, \infty\right)$. This assumption can also be relaxed somewhat, but then the difference in corresponding theorems would be more technical than qualitative. The methods to be employed here will require certain basic observations from classical Lyapunov stability theory for which reference is made to [3], [5]. In Theorem 2, it is shown that the same conclusion holds provided the null solution to the linearization is stable and, of course, the perturbation is small enough. This particular result will be compared to others below.

Finally, it is a nontrivial remark to note that the methods employed here are not necessarily specialized to second order equations. In fact, the usual conversion to a first order system will produce results for certain $n$th order equations. These generalizations will not be detailed here since the main results are to be compared with other known results for second order equations.

2. On asymptotic behavior for (1.1) and (1.2). The following definitions are recalled from [5, p. 54]; see also [3, p. 45], where strong stability is alternately defined for linear systems and is called "restrictive" stability. It is always assumed that $f(t, 0,0)=0$ in (1.2). The null solution is strongly stable if it is (Lyapunov) stable and $\left|x\left(t_{1}\right)\right|+\left|x^{\prime}\left(t_{1}\right)\right| \leqq \varepsilon$ for some $t_{1} \geqq t_{0}$ implies that $|x(t)|+\left|x^{\prime}(t)\right| \leqq \varepsilon$ for all $t \geqq t_{0}$; the null solution is asymptotically stable if it is stable and there exists a $\delta_{0}>0$ such that $\left|x\left(t_{0}\right)\right|+\left|x^{\prime}\left(t_{0}\right)\right|<$ $\delta_{0}$ implies $|x(t)|+\left|x^{\prime}(t)\right|$ tends to zero as $t$ tends to positive infinity.

An easy example in [5] shows that one may have strong stability without asymptotic stability. However, it is obvious from the definitions that the two are always incompatible. This inconsistency will be exploited here to reveal some facts about the asymptotic behavior of solutions to (1.1) and (1.2). First, a useful and well-known lemma is recalled.

Lemma. If the null solution to (1.1) is stable and if there exists $\gamma$ in $L\left[t_{0}, \infty\right)$ such that

$$
|f(t, x, y)| \leqq \gamma(t)[|x|+|y|],
$$

for $(t, x, y)$ in $\left[t_{0}, \infty\right) \times(-\infty,+\infty) \times(-\infty,+\infty)$, then the null solution to (1.2) is strongly stable. 
The proof uses the fact that stability for (1.1) is also strong stability for that equation (see [5, p. 58]). Also, the assumption on $f$ is sufficient to preserve the strong stability from (1.1) to (1.2); this is a corollary to Theorem 7 in $[5$, p. 66]. Thus, under the conditions of the lemma, the Lyapunov stability of the null solution to (1.1) implies that that solution to both (1.1) and (1.2) is strongly stable.

It is now possible to state a result for (1.1); note that there is no mention made of any kind of stability.

THEOREM 1. If $a$ is continuous on $\left[t_{0}, \infty\right)$, then there exists at least one solution $y(t)$ to (1.1) for which

$$
\lim _{t \rightarrow \infty} \sup \left[|y(t)|+\left|y^{\prime}(t)\right|\right]>0 .
$$

Proof. If every solution has the property that $|y(t)|+\left|y^{\prime}(t)\right|$ tends to zero as $t \rightarrow \infty$, then the null solution must be stable and, in fact, asymptotically stable. But, as has been previously remarked, the stability for (1.1) also implies strong stability. This contradicts the fact that a solution cannot be both asymptotically and strongly stable.

Regarding (1.2), it is not difficult to see, using results from [6] or [5], that under conditions which are so stringent as to imply the asymptotic stability of (1.1), it follows that all solutions to (1.2) near zero in fact tend to zero, provided $|f(t, x, y)| \leqq \gamma[|x|+|y|]$, for small enough $\gamma$. On the other hand, under conditions which are so stringent as to imply that each solution to (1.1) is unbounded, it follows that there are no bounded, nontrivial solutions to (2.1). (These facts follow from results which may be found in [5, Chapter 3].) These extreme situations compare with the following.

THEOREM 2. If the null solution to (1.1) is stable and there exists $a \gamma$ in $L\left[t_{0}, \infty\right)$ such that

$$
|f(t, x, y)| \leqq \gamma(t)[|x|+|y|] .
$$

for all $(t, x, y)$ in $\left[t_{0}, \infty\right) \times(-\infty,+\infty) \times(-\infty,+\infty)$, then there exists at least one solution $y(t)$ to $(1.2)$ for which

$$
\lim _{t \rightarrow \infty} \sup \left[|y(t)|+\left|y^{\prime}(t)\right|\right]>0 .
$$

This theorem seems to be an intermediate to those just mentioned. It seems a reasonable conjecture that the theorem is true without the stability assumption.

PROOF. The proof is very brief in view of the above lemma. If each solution $y(t)$ to (1.2) has the property that $|y(t)|+\left|y^{\prime}(t)\right| \rightarrow 0$ as $t \rightarrow \infty$, then 
it would follow that the null solution is both asymptotically and strongly stable, again an impossibility.

\section{REFERENCES}

1. G. Armellini, Sopra una equazione differenziale della dinamica, Rend. Accad. Lincei 21 (1935), 111-116.

2. R. Bellman, Stability theory of differential equations, McGraw-Hill, New York, 1953. MR 15, 794.

3. L. Cesari, Asymptotic behavior and stability problems in ordinary differential equations, 2nd ed., Ergebnisse der Mathematik und ihrer Grenzgebiete, N.F., Band 16, Academic Press, New York; Springer-Verlag, Berlin, 1963. MR 27 \#1661.

4. D. S. Cohen, The asymptotic behavior of a class of nonlinear differential equations, Proc. Amer. Math. Soc. 18 (1967), 607-609. MR 35 \#3163.

5. W. A. Coppel, Stability and asymptotic behavior of differential equations, Heath, Boston, 1965. MR 32 \#7875.

6. - On the stability of ordinary differential equations, J. London Math. Soc. 39 (1964), 255-260. MR 29 \#1393.

7. H. A. Dekleine, A counterexample to a conjecture in second-order linear equations, Michigan Math. J. 17 (1970), 29-32. MR 41 \#5690.

8. E. Hille, Lectures on ordinary differential equations, Addison-Wesley, Reading, Mass., 1969. MR 40 \#2939.

9. A. Meir, D. Willett and J. S. W. Wong, On the asymptotic behavior of the solutions of $x^{\prime \prime}+a(t) x=0$, Michigan Math. J. 14 (1967), 47-52. MR 35 \#463.

10. G. Sansone, Problemi attuali sulla teoria delle equazioni differenziali ordinarie e su alcuni tipi di equazioni alle derivate parziali, Att. Convegno Mat. Roma, 1942, pp. 179-200. MR 9, 36.

11. —- Equazioni differenziali nel campo reale. Vol. 1, 2nd ed., Zanichelli, Bologna, 1948. MR 10, 193.

12. L. Tonelli, Scritti matematici offerti a Luigi Berzolari, 1936, pp. 404-405.

13. A. Wiman, Über die reellen Losungen der linearen Differentialgleichungen zweiter Ordnung, Ark, Mat. Astr. Fys. 12 (1917), 22 pp.

14. — U Uber eine Stabilitatsfrage in der Theorie der linearen Differentialgleichungen, Acta Math. 66 (1936), 121-145.

Department of Mathematics, University of Arizona, Tucson, Arizona 85721 